\title{
Comparison of Spatial Learning Between Male, Female and Ovariectomized Rats
}

Fatemeh Khodabandehloo', Mahmoud Hosseini ${ }^{* *}$, Fatemeh Saffarzadeh ${ }^{3}$, Esmaile Farrokhi ${ }^{4}$, Beheshteh Babazadeh ${ }^{4}$

${ }^{1}$ Department of Physiology, AJA University of Medical Sciences, Tehran, Iran

${ }^{2}$ Neurocognitive Research Center, School of Medicine, Mashhad University of Medical Sciences, Mashhad, Iran

${ }^{3}$ Shefa Neuroscience Research Center, Khatam Alanbia Hospital,Tehran, Iran

${ }^{4}$ Neurogenic Inflammation Research Center, School of Medicine, Mashhad University of Medical Sciences, Mashhad, Iran

\section{A BSTRACT}

Introduction: Sex-dependent changes of behavioral responses in humans and animals has been widely investigated. There are also controversial reports regarding the role of sex hormones in memory and learning. The present study carried out to compare spatial learning of male, female and ovariectomized (OVX) rats in Morris water maze. Materials and Methods: Twenty four rats were divided into 3 groups: 1) male, 2) female, 3) OVX. The animals were ovariectomized under ketamine anesthesia $(150 \mathrm{mg} / \mathrm{kg}$, I.P.). The same procedure as OVX rats was performed on the female and male rats except the wound was closed without removing the ovaries. 8 weeks later, the animals of all groups were tested in Morris Water Maze. The escape latency, traveled path lengh and swimming speed to reach the platform were compared between groups. Results: Time latency in both OVX and male groups was significantly higher than female group . However, path length in OVX group was higher than female group, there was no significant difference in path length between male and female or between male and OVX groups. The swimming speed in male group was lower than female group. Conclusion: It might be concluded that endogenous estrogen has an important role in sex dependent differences of spatial learning.

\section{Key words:}

1. Sex

2. Ovariectomy

3. Spatial Learning

4. Rats

*Corresponding Author: Mahmoud Hosseini

E-mail: hosseinim@mums.ac.ir 
مقايسةُ يادَيرى فضايى موشهاى صحر ايیى نر، ماده و اواركتومى شده

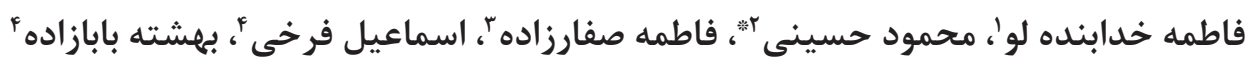

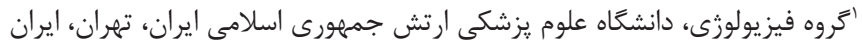

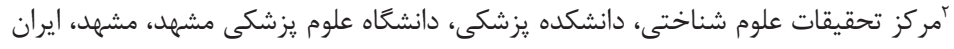

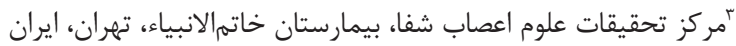

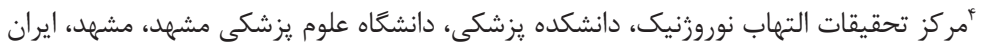

كليد وازهها:

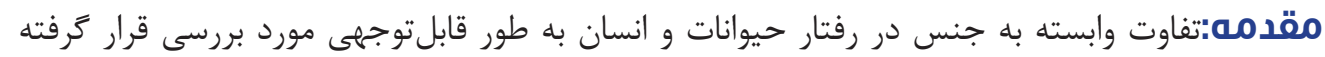

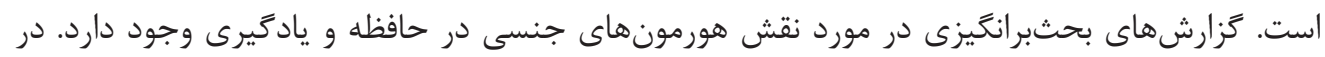

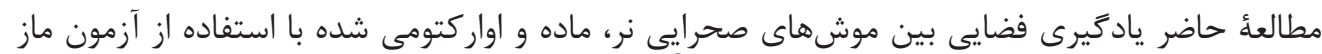

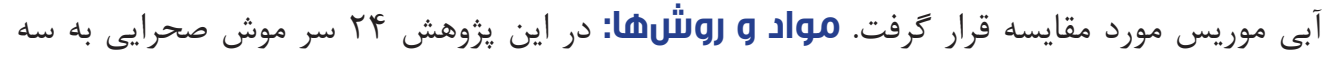

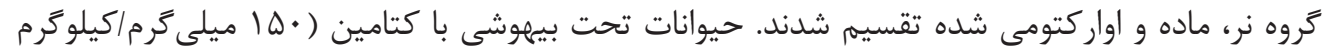

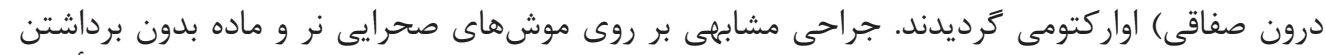

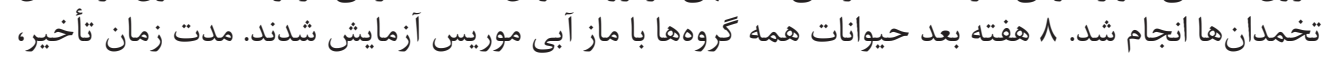

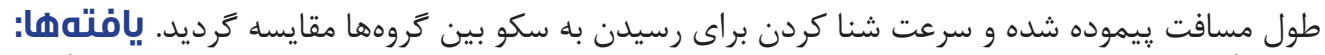

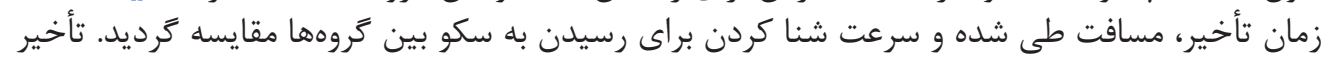

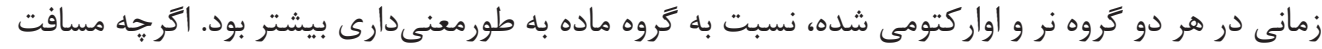

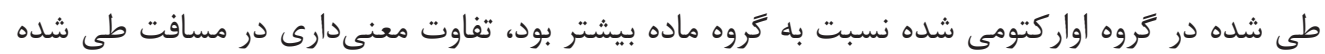

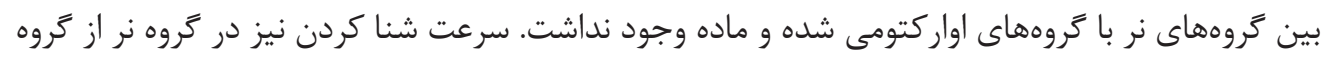

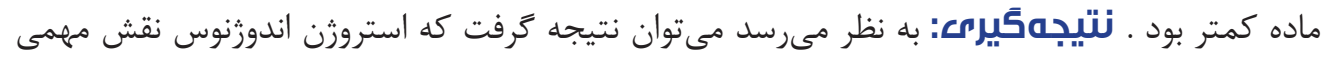

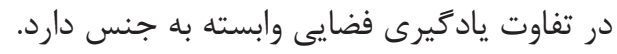

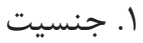

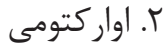
r.

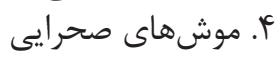

" نويسنده مسئول: سيد محمود حسينى : آدرس الكترونيكى: بوسينده مينول:mums.ac.ir 


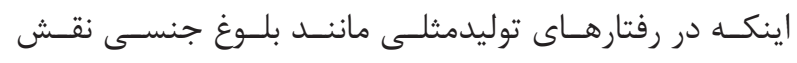

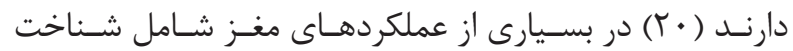

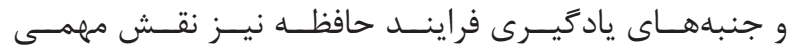

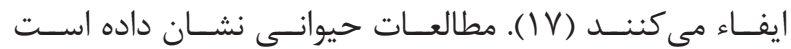

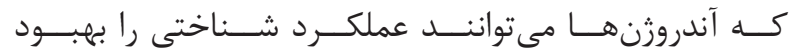

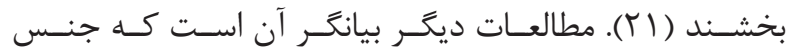

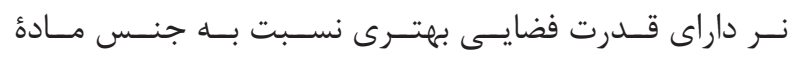

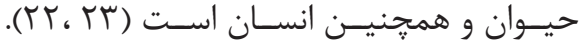

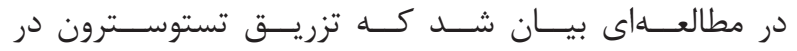

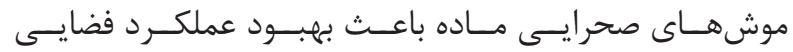

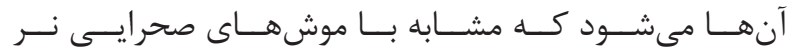

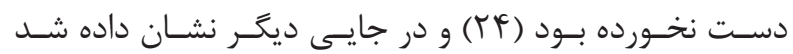

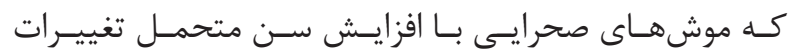

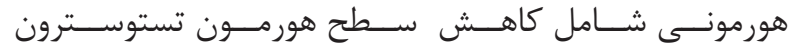

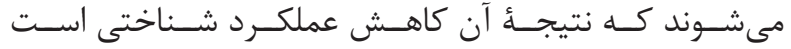

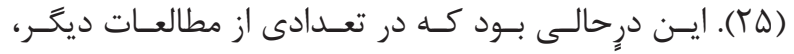

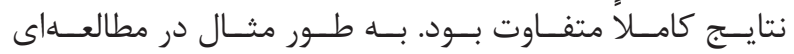

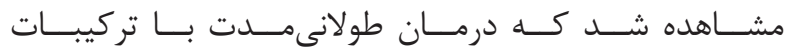

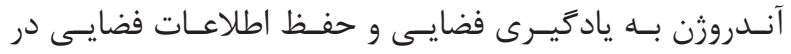

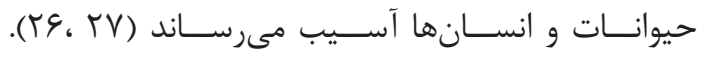

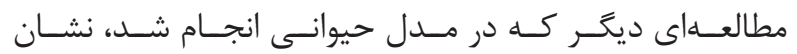

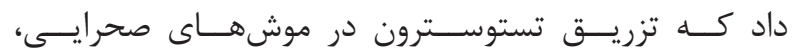

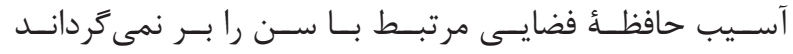

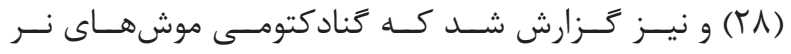

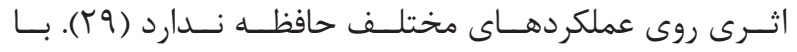

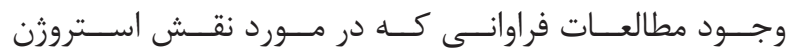

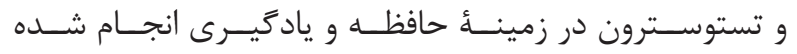

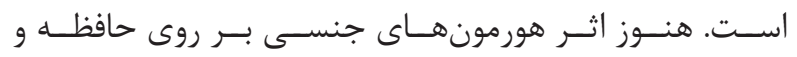

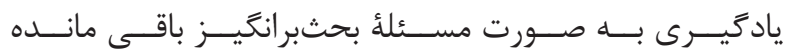

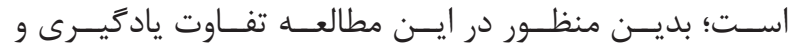

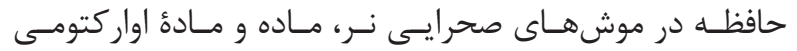

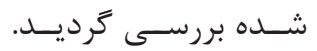

مواد و روشها حيوانات و گروههاى مورد آزمايش

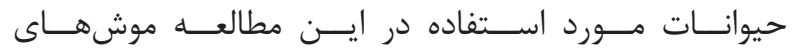

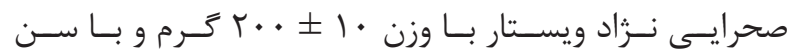

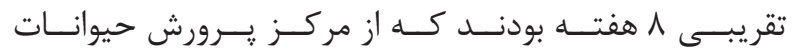

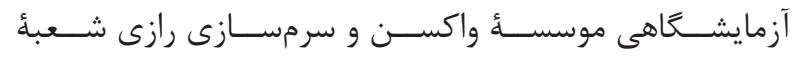

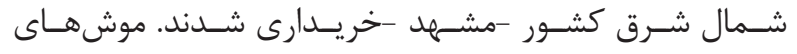

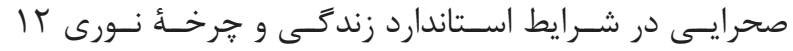

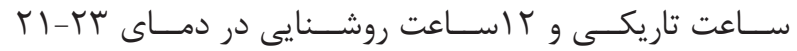

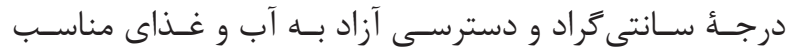

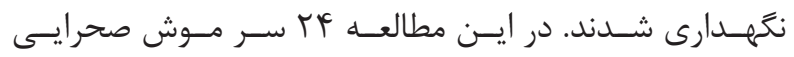

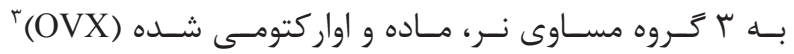

مقدمه

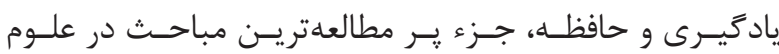

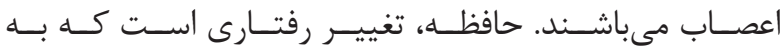

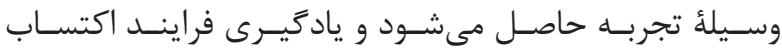

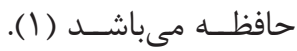
نقـش اسـتروئيدهاى جنسـى در تكامـل نواحيـى از مغـز كه در

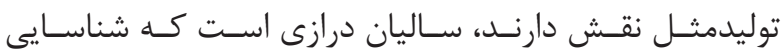

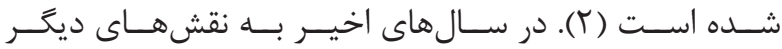

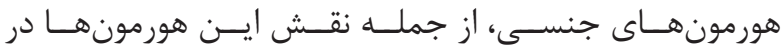

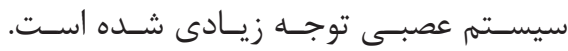

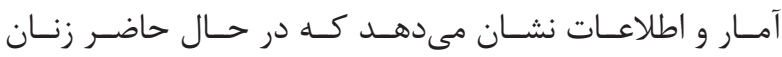

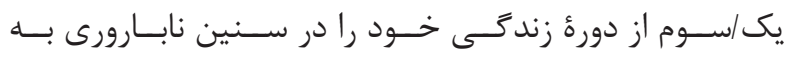

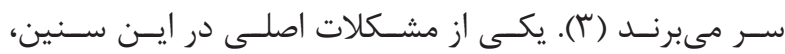

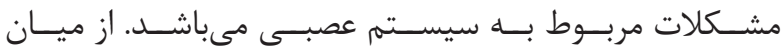

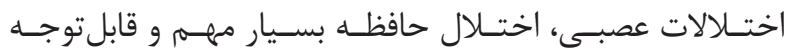

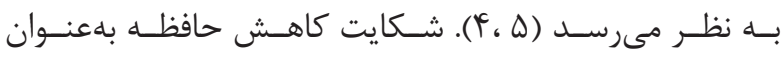

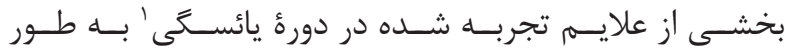

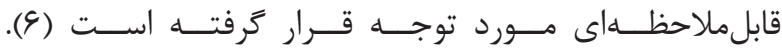

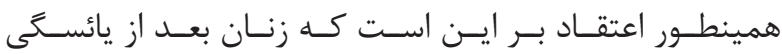

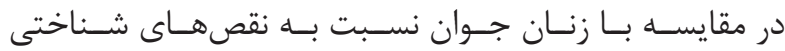

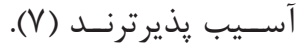

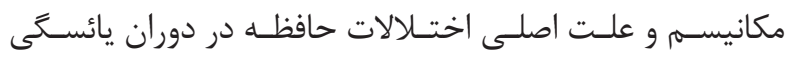

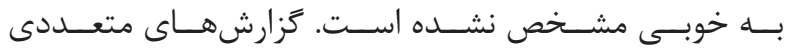

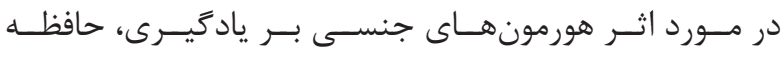

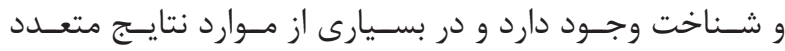

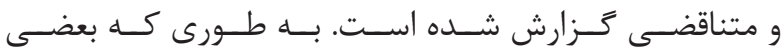

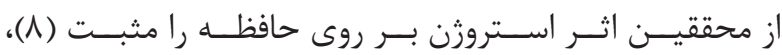

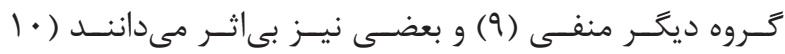

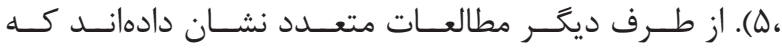

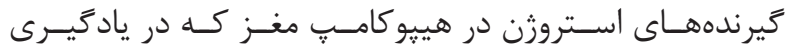

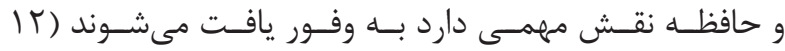

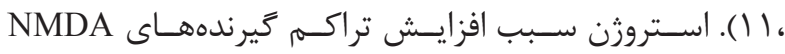

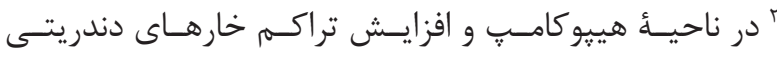

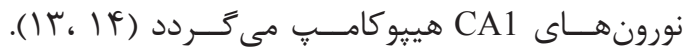

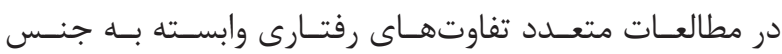

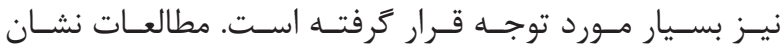

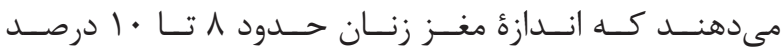

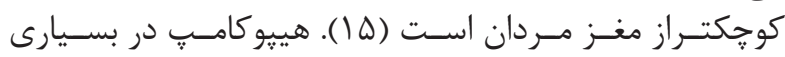

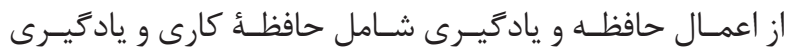

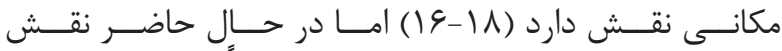

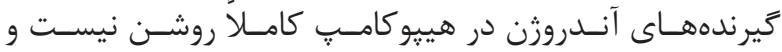

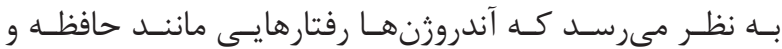

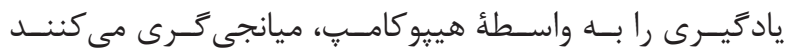

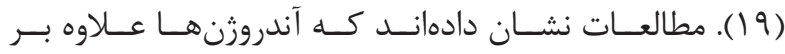

\footnotetext{
${ }^{1}$ Menopause

${ }^{2} \mathrm{~N}$-methyl-d-aspartate

${ }^{3}$ Ovariectomized
} 


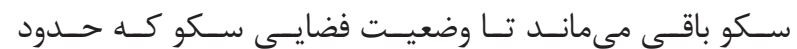

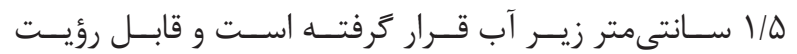

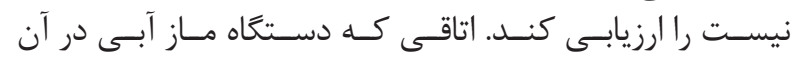

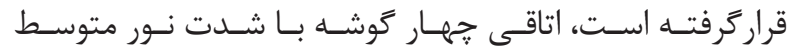

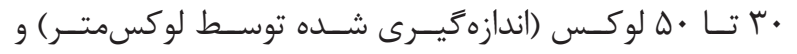

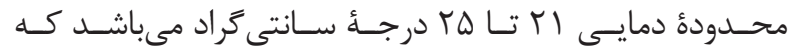

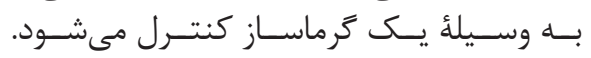

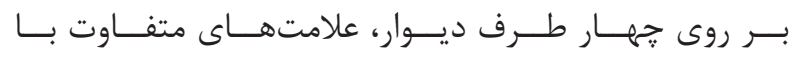

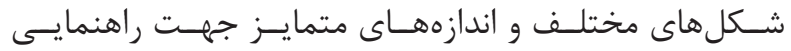

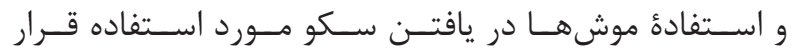

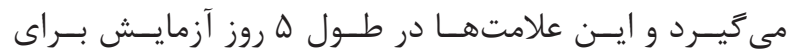

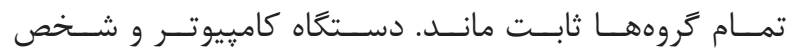

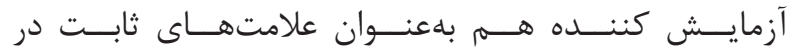

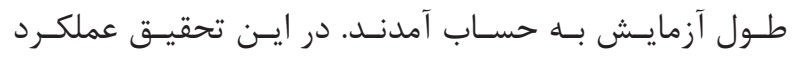

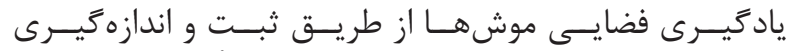

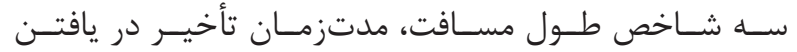

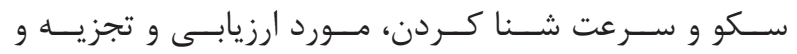

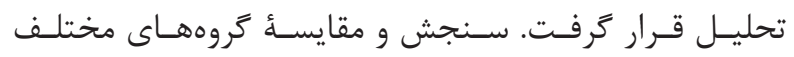

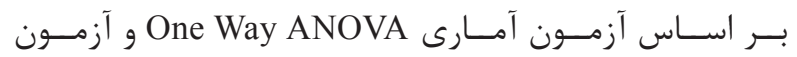

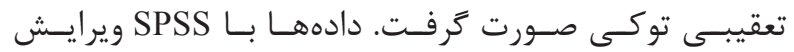

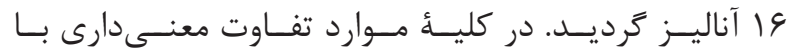

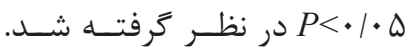

بافتهها

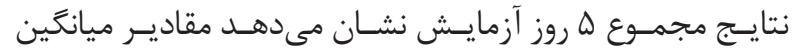

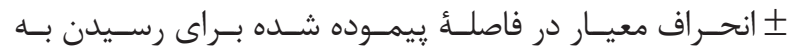

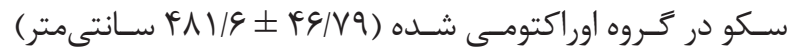

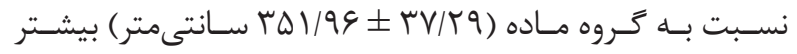

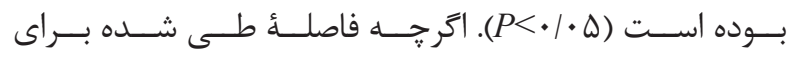

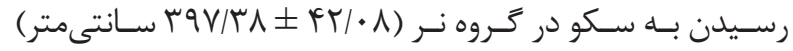

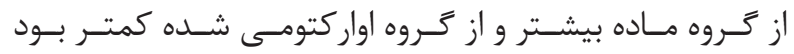
ولـى اختـلاف معنـى دارى نداشـتـند (نمـودار ().

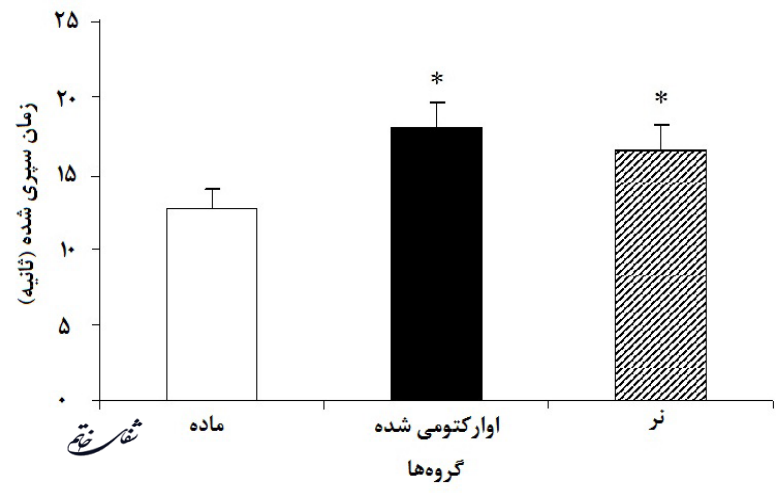

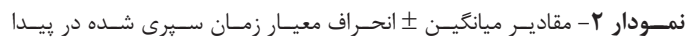

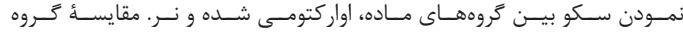

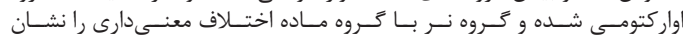

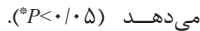

${ }^{4}$ Morris water maze

${ }^{5}$ Path length

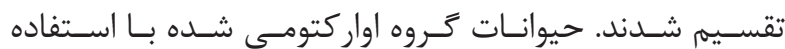

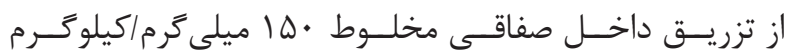

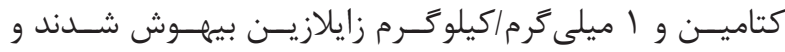

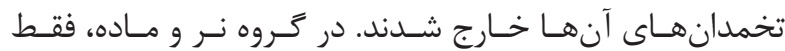

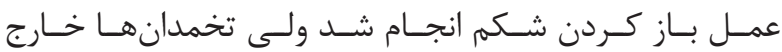

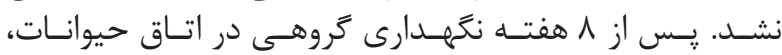

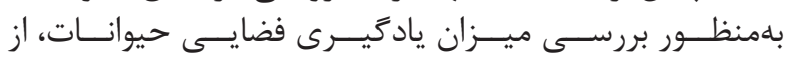

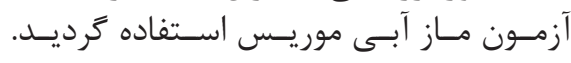

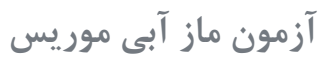

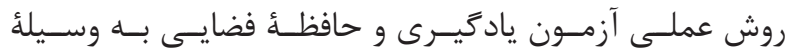

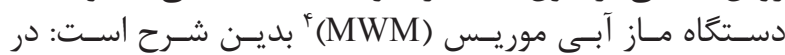

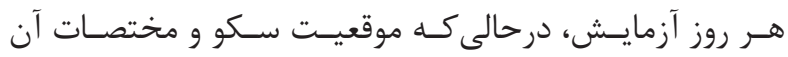

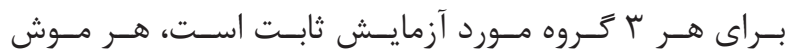

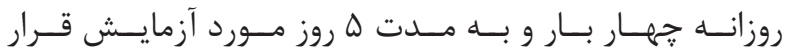

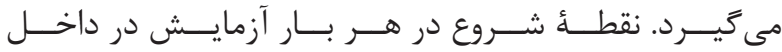

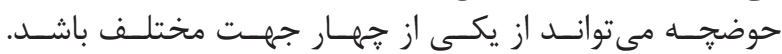

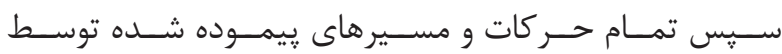

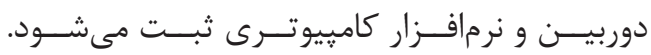

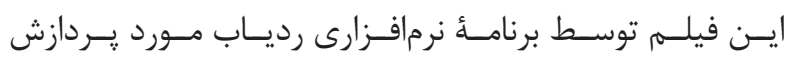

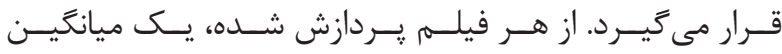

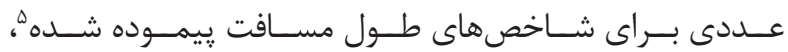

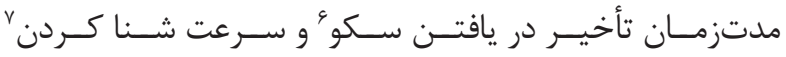

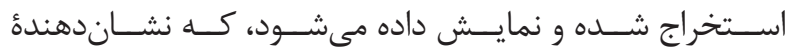

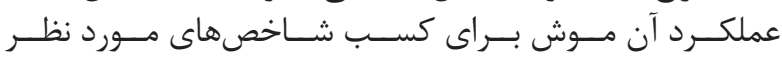

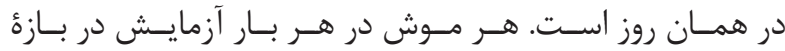

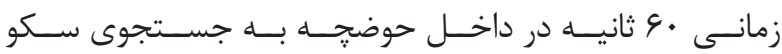

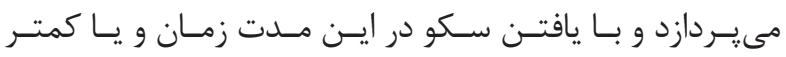

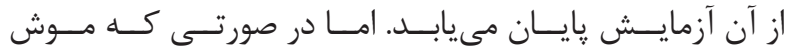

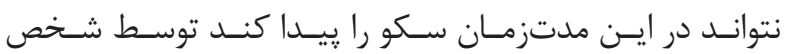

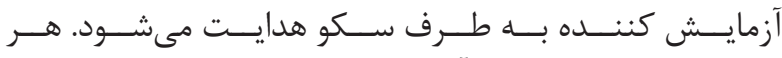

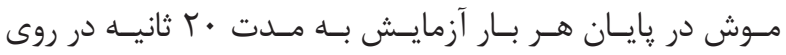

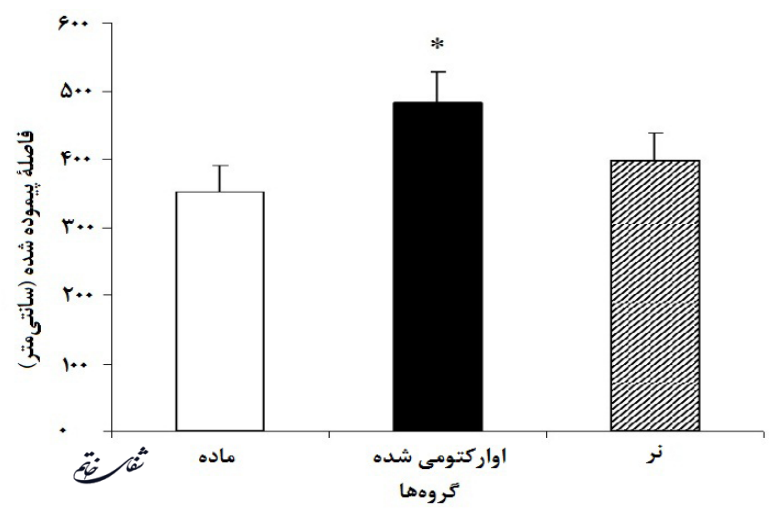

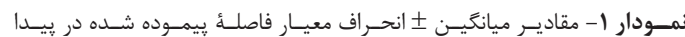

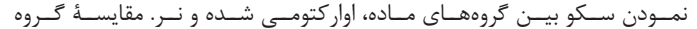

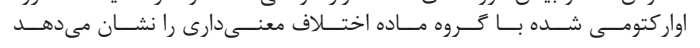
$\left({ }^{*} P<\cdot / \cdot \Delta\right)$

${ }^{7}$ Swimming speed 


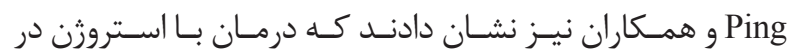

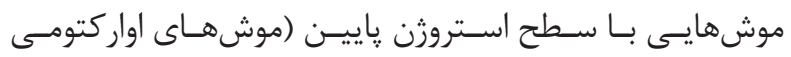

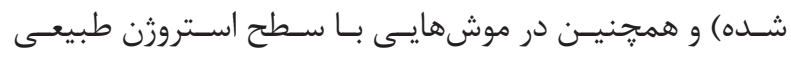

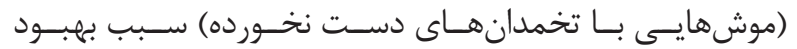

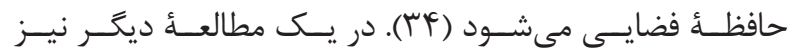

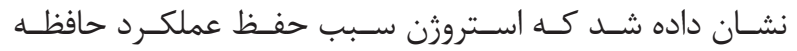

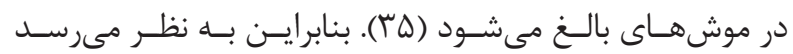

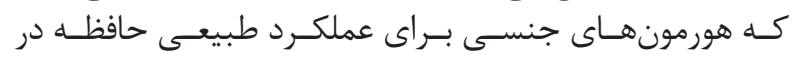

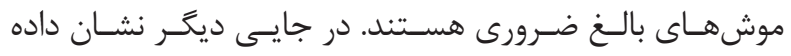

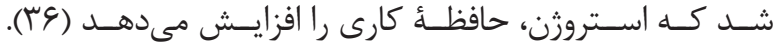

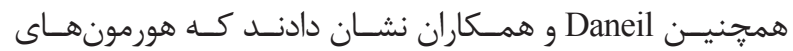

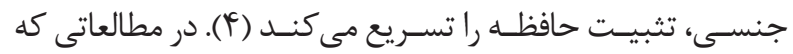

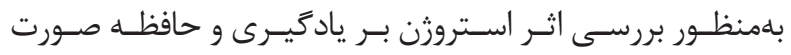

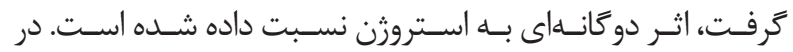

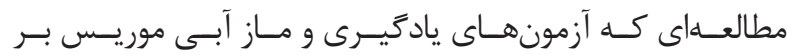

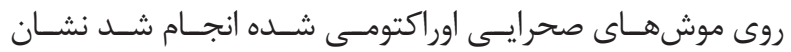

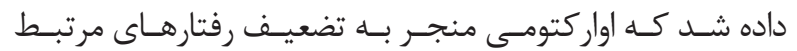

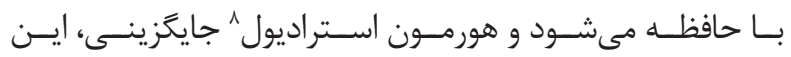

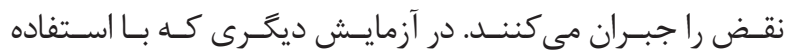

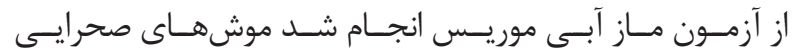

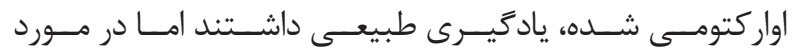

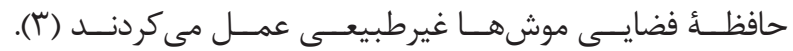

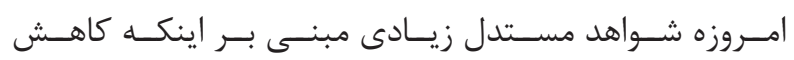

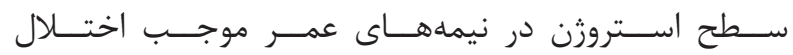

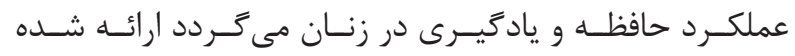

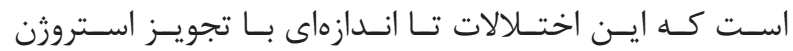

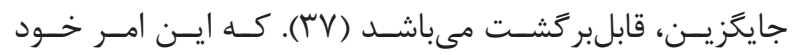

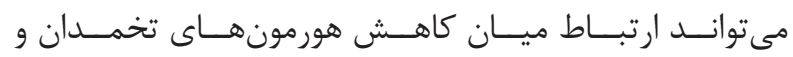

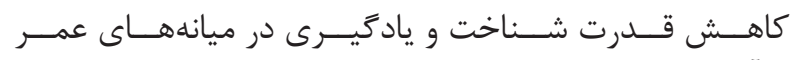

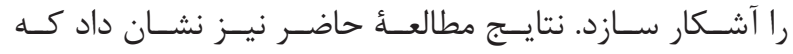

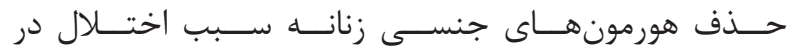

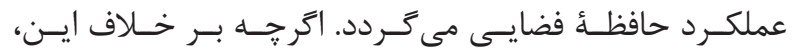

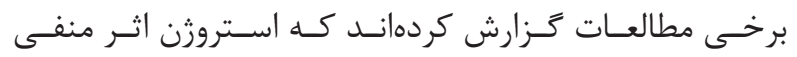

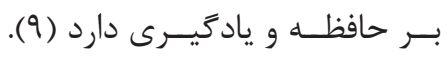

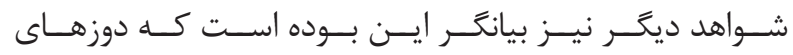

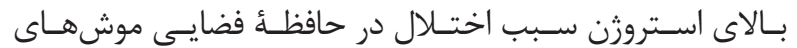

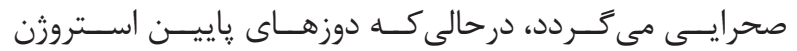

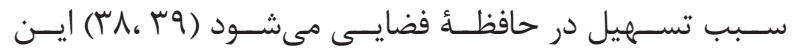

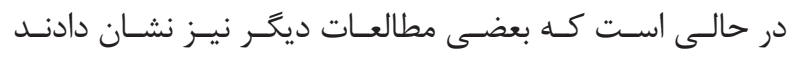

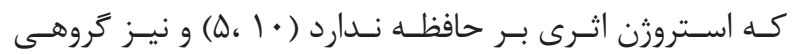

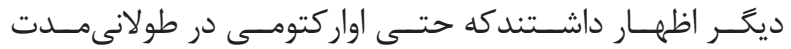

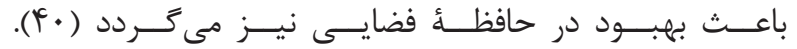

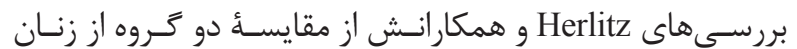

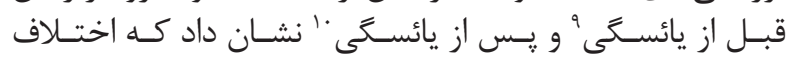

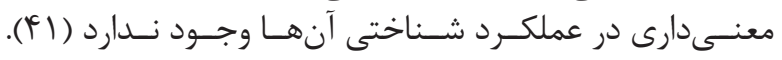

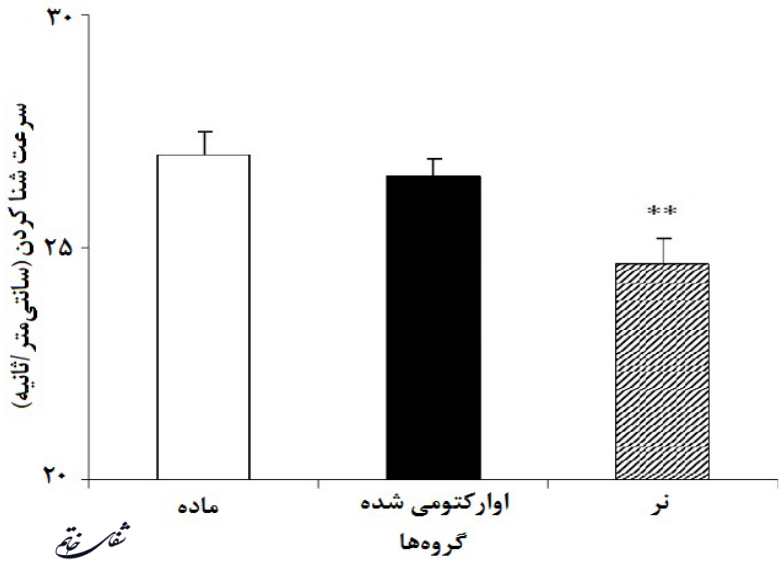

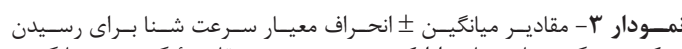

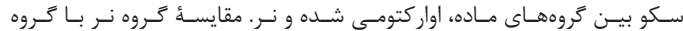

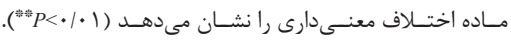

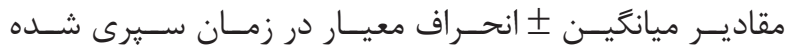

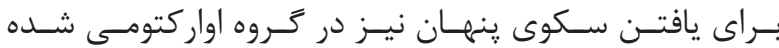

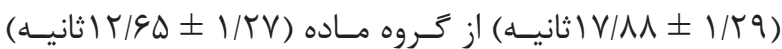

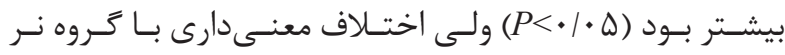

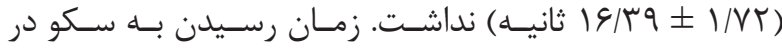

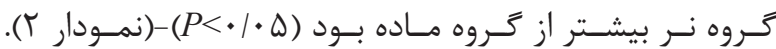

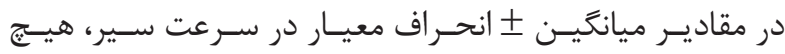

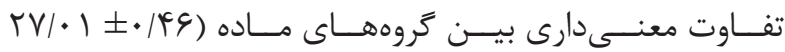

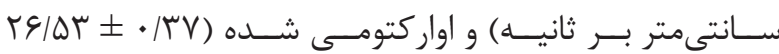

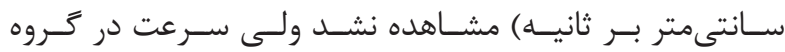

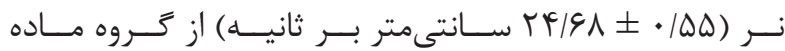

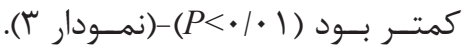
بحث و نتيجه كيرى

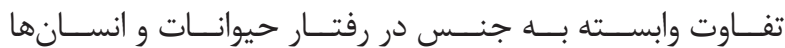

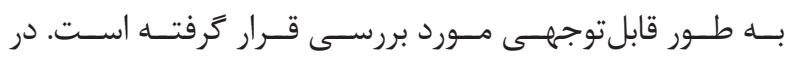

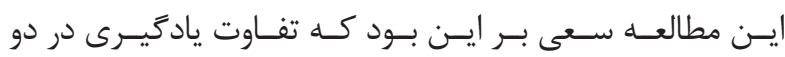

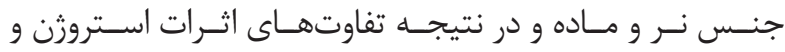

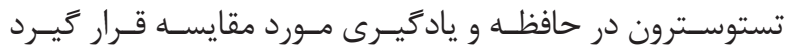

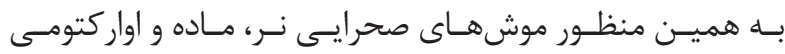

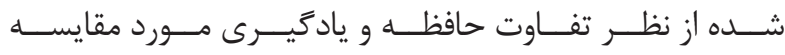

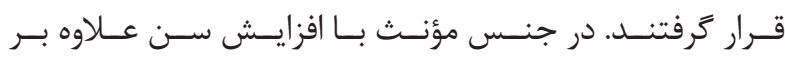

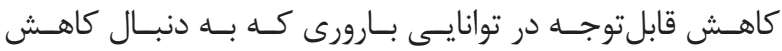

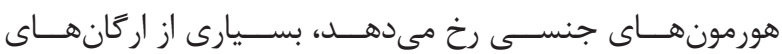

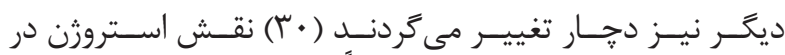

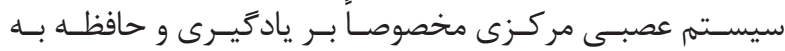

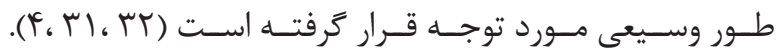

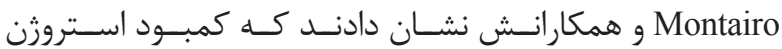

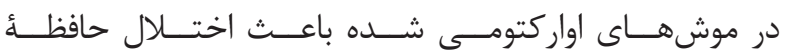

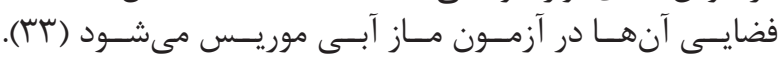

${ }^{8}$ Estradiol

${ }^{9}$ Premenopausal

${ }^{10}$ Postmenopausal 


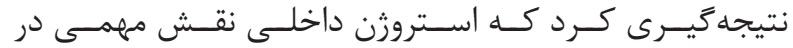

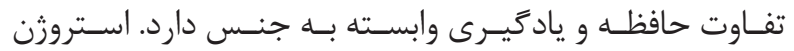

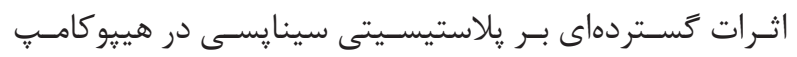

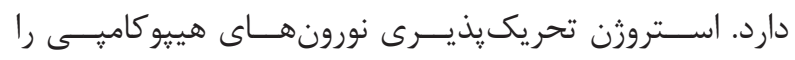

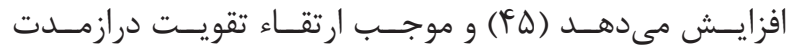

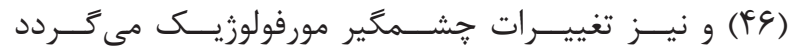

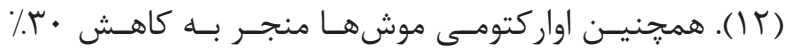

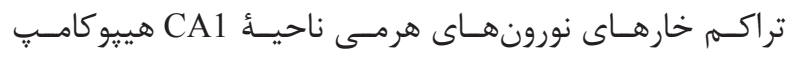

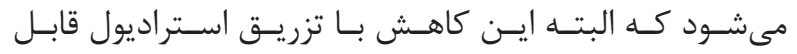

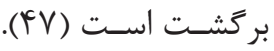

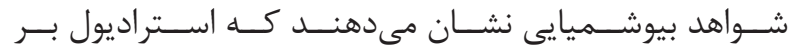

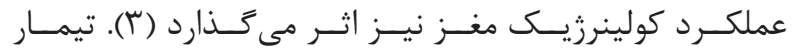

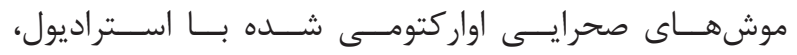

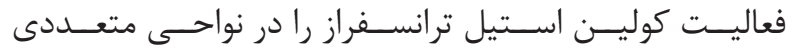

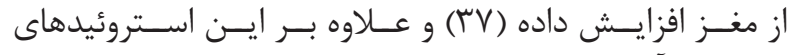

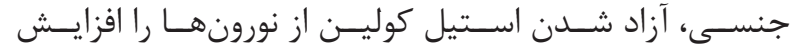

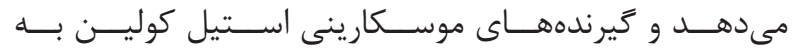

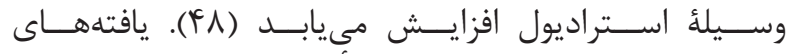

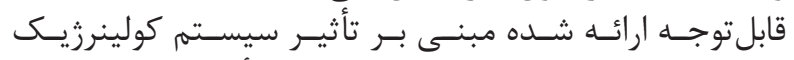

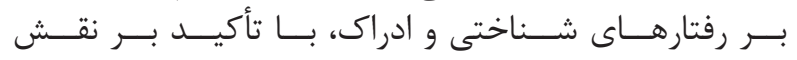

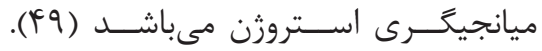

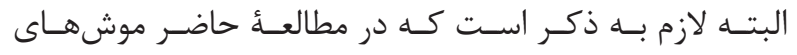

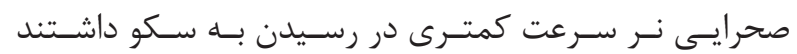

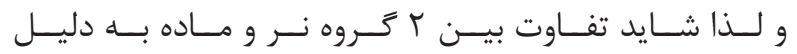

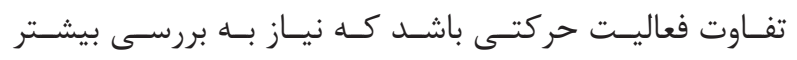

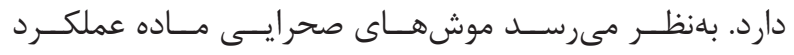

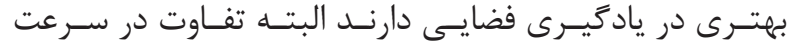

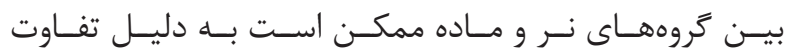

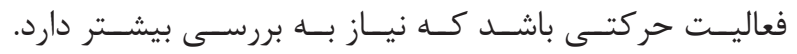

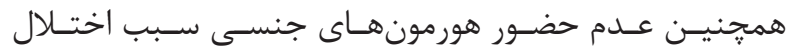

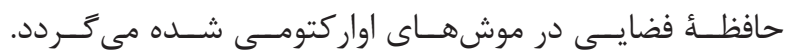

1. Okano H, Hirano T, Balaban E. Learning and memory. Proc Natl Acad Sci U S A. 2000; 97(23): 12403-4.

2. Schantz SL, Widholm JJ. Cognitive effects of endocrine-disrupting chemicals in animals. Environ Health Perspect. 2001; 109(12): 1197-206.

3. Simpkins JW, Green PS, Gridley KE, Singh M, de Fiebre NC, Rajakumar G. Role of estrogen replacement therapy in memory enhancement and the prevention of neuronal loss associated with Alzheimer's disease. Am J Med. 1997; 103(3): 19S-25S.

4. Daniel JM, Fader AJ, Spencer AL, Dohanich GP. Estrogen enhances performance of female rats during

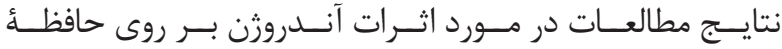

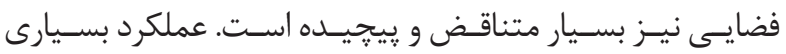

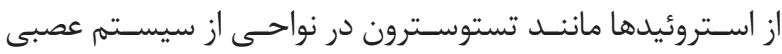

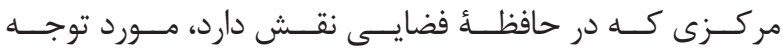

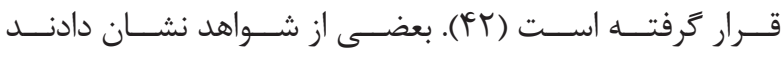

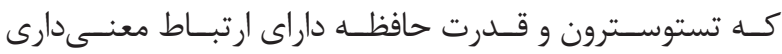

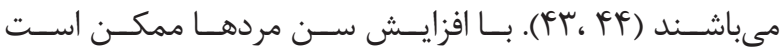

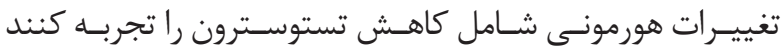

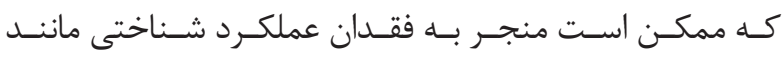

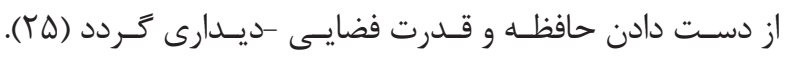

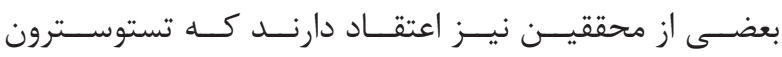

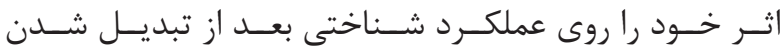

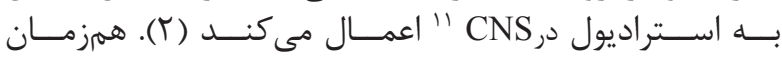

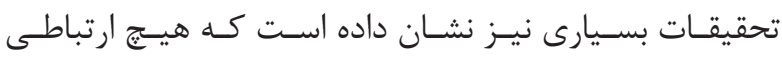

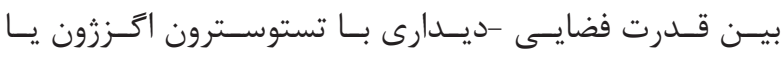

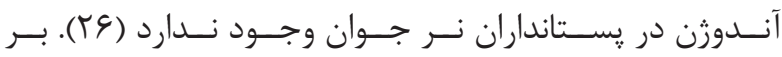

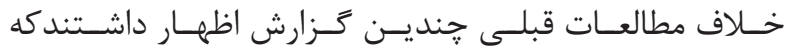

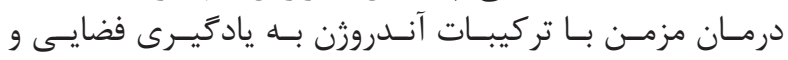

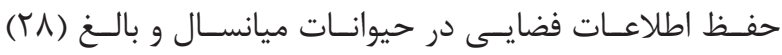

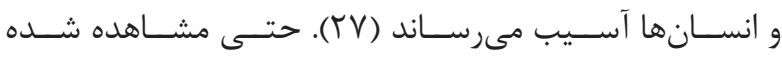

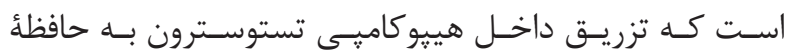

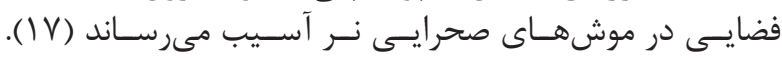

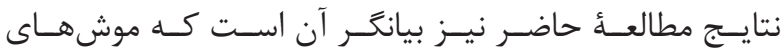

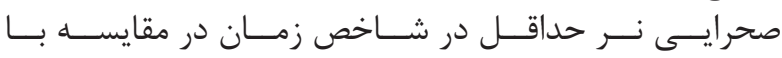

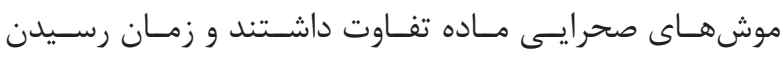

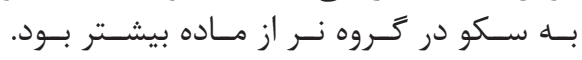

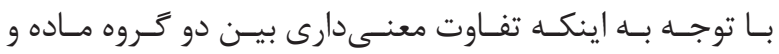

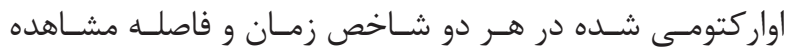

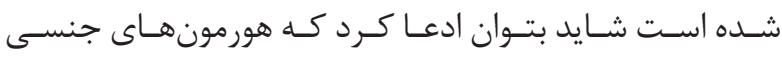

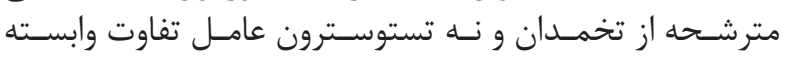

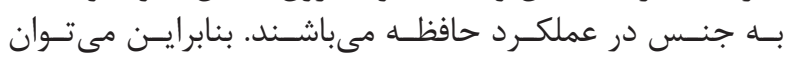

منابع

acquisition of a radial arm maze. Horm Behav. 1997; 32(3): 217-25.

5. Fader AJ, Johnson PE, Dohanich GP. Estrogen improves working but not reference memory and prevents amnestic effects of scopolamine on a radial-arm maze. Pharmacol Biochem Behav. 1999; 62(4): 711-7.

6. Henderson VW. Cognitive changes after menopause: influence of estrogen. Clin obstet gynecol. 2008; 51(3): 618-26.

7. Henderson VW. Aging, estrogens, and episodic memory in women. Cognit Behav Neurol. 2009; 22(4): 205-14.

${ }^{11}$ Central nervous system 
8. O’Neal MF, Means LW, Poole MC, Hamm RJ. Estrogen affects performance of ovariectomized rats in a two-choice water-escape working memory task. Psychoneuroendocrinology. 1996; 21(1): 51-65.

9. Chesler EJ, Juraska JM. Acute administration of estrogen and progesterone impairs the acquisition of the spatial Morris water maze in ovariectomized rats. Horm Behav. 2000; 38(4): 234-42.

10. Healy SD, Braham SR, Braithwaite VA. Spatial working memory in rats: no differences between the sexes. Proc Biol Sci. 1999; 266(1435): 2303-8.

11. Cordoba Montoya D, Carrer H. Estrogen facilitates induction of long term potentiation in the hippocampus of awake rats. Brain Res. 1997; 778(2): 430-8.

12. Woolley CS, McEwen BS. Estradiol regulates hippocampal dendritic spine density via an N-methylD-aspartate receptor-dependent mechanism. J Neurosci. 1994; 14(12): 7680-7.

13. Gazzaley AH, Weiland NG, McEwen BS, Morrison JH. Differential regulation of NMDAR 1 mRNA and protein by estradiol in the rat hippocampus. J Neurosci. 1996; 16(21): 6830-8.

14. McEwen B. Estrogen actions throughout the brain. Recent Prog Horm Res. 2002; 57: 357-84.

15. Goldstein JM, Seidman LJ, Horton NJ, Makris N, Kennedy DN, Caviness VS, et al. Normal sexual dimorphism of the adult human brain assessed by in vivo magnetic resonance imaging. Cereb Cortex. 2001; 11(6): 490-7.

16. Hojo Y, Hattori TA, Enami T, Furukawa A, Suzuki K, Ishii HT, et al. Adult male rat hippocampus synthesizes estradiol from pregnenolone by cytochromes P45017alpha and P450 aromatase localized in neurons. Proc Natl Acad Sci USA. 2004; 101(3): 865-70.

17. Naghdi N, Nafisy N, Majlessi N. The effects of intrahippocampal testosterone and flutamide on spatial localization in the Morris water maze. Brain Res. 2001; 897(1): 44-51

18. Kimoto T, Tsurugizawa T, Ohta Y, Makino Jy, Tamura H-o, Hojo Y, et al. Neurosteroid synthesis by cytochrome p450-containing systems localized in the rat brain hippocampal neurons: N-methyl-D-aspartate and calcium-dependent synthesis. Endocrinology. 2001; 142(8): 3578-89.

19. Williams CL, BarnettAM, Meck WH. Organizational effects of early gonadal secretions on sexual differentiation in spatial memory. Behav Neurosci. 1990; 104(1): 84-97.
20. Wilson JD. The role of androgens in male gender role behavior. Endocr Rev. 1999; 20(5): 726-37.

21. Frye CA, Edinger KL, Seliga AM, Wawrzycki JM. 5alpha-reduced androgens may have actions in the hippocampus to enhance cognitive performance of male rats. Psychoneuroendocrinology. 2004; 29(8): 1019-27.

22. Nakamura N, Fujita H, Kawata M. Effects of gonadectomy on immunoreactivity for choline acetyltransferase in the cortex, hippocampus, and basal forebrain of adult male rats. Neuroscience. 2002; 109(3): 473-85

23. Williams CL, Meck WH. The organizational effects of gonadal steroids on sexually dimorphic spatial ability. Psychoneuroendocrinology. 1991; 16(1): 155-76.

24. Isgor C, Sengelaub DR. Prenatal gonadal steroids affect adult spatial behavior, CA1 and CA3 pyramidal cell morphology in rats. Horm Behav. 1998; 34(2): 183-98.

25. Tan R. Memory loss as a reported symptom of andropause. Arch Androl. 2001; 47(3): 185-9.

26. Galea LA, Kavaliers M, Ossenkopp K-P, Hampson E. Gonadal hormone levels and spatial learning performance in the Morris water maze in male and female meadow voles, Microtus pennsylvanicus. Horm Behav. 1995; 29(1): 106-25.

27. Gouchie C, Kimura D. The relationship between testosterone levels and cognitive ability patterns. Psychoneuroendocrinology. 1991; 16(4): 323-34.

28. Goudsmit E, Van de Poll N, Swaab D. Testosterone fails to reverse spatial memory decline in aged rats and impairs retention in young and middle-aged animals. Behav Neural Biol. 1990; 53(1): 6-20.

29. Van Hest A, Van Kempen M, Van Haaren F, Van de Poll NE. Memory in male and female Wistar rats: Effects of gonadectomy, and stimulus presentations during the delay interval. Behav Brain Res. 1988; 29(1): 103-10. 30. Nelson HD. Menopause. Lancet. 2008; 371(9614): 760-70.

31. Woolley CS. Estrogen-mediated structural and functional synaptic plasticity in the female rat hippocampus. Horm Behav. 1998; 34(2): 140-8.

32. Harman D. Free radical theory of aging: an update. Ann NY Acad Sci. 2006; 1067(1): 10-21.

33. Monteiro SC, Stefanello FM, Vianna LP, Matté C, Barp J, Belló-Klein A, et al. Ovariectomy enhances acetylcholinesterase activity but does not alter ganglioside content in cerebral cortex of female adult rats. Metab Brain Dis. 2005; 20(1): 35-44. 
34. Ping SE, Trieu J, Wlodek ME, Barrett GL. Effects of estrogen on basal forebrain cholinergic neurons and spatial learning. J neurosci Res. 2008; 86(7): 1588-98.

35. El-Bakri NK, Islam A, Zhu S, Elhassan A, Mohammed A, Winblad B, et al. Effects of estrogen and progesterone treatment on rat hippocampal NMDA receptors: relationship to Morris water maze performance. J Cell Mol Med. 2004; 8(4): 537-44.

36. Pompili A, Tomaz C, Arnone B, Tavares MC, Gasbarri A. Working and reference memory across the estrous cycle of rat: a long-term study in gonadally intact females. Behav Brain Res. 2010; 213(1): 10-8.

37. Hogervorst E, Boshuisen M, Riedel W, Willeken C, Jolles J. The effect of hormone replacement therapy on cognitive function in elderly women. Psychoneuroendocrinology. 1999; 24(1): 43-68.

38. Holmes MM, Wide JK, Galea LA. Low levels of estradiol facilitate, whereas high levels of estradiol impair, working memory performance on the radial arm maze. Behav Neurosci. 2002; 116(5): 928-34.

39. Luine VN, Richards ST, Wu VY, Beck KD. Estradiol enhances learning and memory in a spatial memory task and effects levels of monoaminergic neurotransmitters. Horm Behav. 1998; 34(2): 149-62.

40. Bimonte-Nelson HA, Singleton RS, Hunter CL, Price KL, Moore AB, Granholm A-CE. Ovarian hormones and cognition in the aged female rat: I. Longterm, but not short-term, ovariectomy enhances spatial performance. Behav Neurosci. 2003; 117(6): 1395-406.

41. Herlitz A, Thilers P, Habib R. Endogenous estrogen is not associated with cognitive performance before, during, or after menopause. Menopause. 2007; 14(3): 425-31.
42. Barrett-Connor E, Goodman-Gruen D, Patay B. Endogenous Sex Hormones and Cognitive Function in Older Men 1. J Clin Endocrinol Metab. 1999; 84(10): 3681-5.

43. Alexander GM, Packard MG, Hines M. Testosterone has rewarding affective properties in male rats: implications for the biological basis of sexual motivation. Behav Neurosci. 1994; 108(2): 424-8.

44. Flood JF, Roberts E. Dehydroepiandrosterone sulfate improves memory in aging mice. Brain Res. 1988; 448(1): 178-81.

45. Wong M, Moss RL. Long-term and short-term electrophysiological effects of estrogen on the synaptic properties of hippocampal CA1 neurons. J Neuroscience. 1992; 12(8): 3217-25.

46. Good M, Day M, Muir JL. Cyclical changes in endogenous levels of oestrogen modulate the induction of LTD and LTP in the hippocampal CA1 region. Eur J Neuroscience. 1999; 11(12):4476-80.

47. Gould E, Woolley CS, Frankfurt M, McEwen BS. Gonadal steroids regulate dendritic spine density in hippocampal pyramidal cells in adulthood. J Neuroscience. 1990; 10(4): 1286-91.

48. Mitsushima D, Takase K, Takahashi T, Kimura F. Activational and Organisational Effects of Gonadal Steroids on Sex-Specific Acetylcholine Release in the Dorsal Hippocampus. J Neuroendocrinology. 2009; 21(4): 400-5.

49. Tinkler GP, Voytko ML. Estrogen modulates cognitive and cholinergic processes in surgically menopausal monkeys. Prog Neuro-psychopharmacolBiol Psychiatry. 2005; 29(3): 423-31. 